\title{
Coding Self Representations: Linking Postural Dynamics to Egocentric Tuning
}

\author{
Denis Larrivee* \\ Mind and Brain Institute, University of Navarra Medical School, Spain and Loyola University Chicago, USA
}

*Corresponding author: Denis Larrivee, Mind and Brain Institute, University of

Navarra Medical School, Spain and Loyola University Chicago, USA.

Received Date: January 08, 2019

Published Date: January 29, 2019

\begin{abstract}
The concept of the neural representation of the self, evoked when the body is dynamically engaged in intentional action, is traced to the notion of the motor image. Insights drawn from the motor image posit that bodily representation is a key feature stabilizing individual motions of the plan as teleologically situated, that is, linking an agent to an objective terminus. Current work is beginning to disentangle representational content that is globally attuned to the whole individual from that for discrete movements. These studies suggest that representations of body posture are likely to be an important proxy for global self-representation in dynamic actions and may be directly modified by goal specific content. Candidate influences for goal related modulation are likely to include egocentric coding in the posterior parietal and premotor cortices that shape self representations to yield goal directed motor movement.
\end{abstract}

Keywords: Motor image; Predictive processing; Postural tuning; Neural coding; Corollary discharge; Body schema; Egocentric; Allocentric

\section{Introduction}

The modern concept of the neural representation of the self, evoked in circumstances where the body is dynamically engaged in intentional actions, has evolved from several experimental legacies traced to the notion of the motor image [1-3]. This image is so designated to indicate a covert action undertaken only mentally and as a simulation of a non-executed action. In its current understanding the motor image represents the feature elements of an intended motor trajectory, that is, the projected series of motions that will be executed in a motor plan. Accordingly, it contains the signal features needed for plan execution [4].

Insights from the motor image posit that bodily representation is a key feature that stabilizes individual motions of the plan as teleologically situated; that is, one where inscribed actions are bound to an agent having an objective destination. Afferent input used to generate the body representation, termed the body schema, has been shown to originate from somatotopic and interoceptive input. Accordingly, the motor image includes not only the discrete movements of bodily limbs but also their association with the representation of the whole body; that is, discrete movements are

contextualized with respect to the whole individual, who is neurally represented by his body. Neural representations contained in the motor image thus include those of the body schema, specific limb movements, and the target, understood to be the intended goal of actions that are undertaken [5]. Actions are therefore planned in the context of the whole individual with respect to their intended outcome [6,7], how the motor image is structured and implemented, accordingly, has substantial bearing on the optimization of motor performance.

Studies of the image reveal that its feature elements include distinct and reciprocal contributions from central and peripheral origins; accordingly, they underscore the essential unity of dynamic performance even in its covert formulation. Central influences have been classically demonstrated by the presence of movements undertaken in the absence of sensorial input. Lashley notably observed that humans and animals were capable of motor actions despite the loss of afferent input. Later experiments in monkeys also confirmed a central origin by showing that deafferentation of spinal dorsal motor roots [8] did not prevent pointing movements. 
When the original relationship between a movement and its spatiotemporal origin had been altered, however, limb movements failed to correspond to their expected trajectories, involving instead a mis reaching followed by subsequent compensatory movements. Taken together, these results indicated that initial movements responded to memorized, centrally evoked motor commands that were sent to the sensory cortex, termed efference copy or corollary discharges, but subsequent movements required sensory feedback to correctly maintain their targeting.

In continuous motions, feedback from sensory cues is therefore essential to motor execution, where it is coupled with central commands in a mutually reciprocal and sustained process designated a forward projection model [9]. This coupling is necessary since as the body undergoes motion, its spatiotemporal position is continually changing; so also, the sensory cues that reference it. In consequence, peripheral contributions provide an ongoing stream of sensory updating that adjusts motor execution, a phenomenon that has been likened to a perception action loop [10]. Its significance has been demonstrated in many studies, including investigations of the body's dynamical interaction with the environment in developing infants, acquired motor abilities, biodynamic studies of movement and proprioception related to perception, and in the motoric encoding of actions such as reaching [11-13].

Critically, the need to interact with the world identifies the individual as a 'unit of interaction', as Francisco Varela noted. Representation of this unit thus requires that it be globally represented. The self percept, accordingly, is not an abstraction but a representation emerging from the body as a whole that identifies it as a spatiotemporal reality needed to confer unity for performance and other ends [14]. Its interactive character thus situates the self as a unitary dynamic; hence, the representation of self is continually evolving as its spatial coordinates change through time. Contiguous dynamics of discrete limb movements must therefore be sited in the context of this mobile frame to be understood as belonging to the self.

It is currently unknown which representations signify the self or how these relate to individual actions of the motor image. However, recent studies have revealed that representations of bodily posture may act as proxies for global self representation [15]. Evidence from schizophrenic patients, moreover, has revealed that two levels of self-recognition exist, one automatic that is used for action identification and a second, conscious and employed for carrying out intentional actions. In these patients intentional, but not automatic attributable actions are affected, indicating that the patients are unable to attribute intentional actions to the selfrepresentation [16]. By extension, postural content may be modified in the construction of the motor plan when inscribing intentional actions that incorporate goal-specific outcomes. Accordingly, this paper will review evidence that postural representations frame discrete actions and that they also incorporate information about specific intentions, that is, they frame individual actions of motor planning as teleologically situated.

\section{Methods}

Literature reviews were conducted using articles taken from PubMed, EBSCOhost, and SpringerLink data bases and demarcated by the keywords motor image, neural representation, predictive processing, schizophrenia, body schema, corollary discharge, free energy principle, egocentric and allocentric circuits, spatial navigation, and postural tuning. Articles directed to neural representation of the motor image, factors affecting motor planning, medical lesions of the motor image entailed in psychiatric disorders, and self representation were specifically chosen for inclusion in the review.

\section{Evoking Corporal Representations}

A key and consistent finding in varied self representations is the association between the self and a representation of the whole body that is physically realized in the neural activity of the brain. Disturbances of self observed in the schizophrenia patient, especially, have been shown to include an abnormal sense of body ownership, loss of ego boundary, or a confused sense of action attribution, each a property that relates to the body $[17,18]$. Young infants, moreover lack mechanisms needed for situating the motor plan with respect to the self as represented in the whole body [5]. For example, in developing toddlers discrepancies between explicit and implicit formats in navigational false-belief tests display an inability to mentally construct a motor trajectory by situating oneself with respect to the task at hand, consistent with results obtained in the A not B task [6]. In other words, it is necessary to conceive of the self through the performing body in order to formulate a motor plan, an ability lacking in very young children.

Expressed through body ownership the sense of self evokes both the physical reality of the self and the personal possession of the self. It can occur, therefore, whether bodily actions are undertaken by oneself or mediated by another through the body. In action attribution, for instance, the perception that the body is the source of its own actions links the self-percept to actions undertaken for the good of the whole individual. This experiential consistency in varying dynamical contexts constitutes strong evidence of the need to frame the self as a bodily representation.

Philosophically, the need to instantiate the self within the framework of the body can be understood as an a priori condition required for autonomous existence. Goal directed actions, especially, acquire intelligibility only if performed to secure an end for the whole individual. Accordingly, actions undertaken for the good of the whole must define it as an objective physical reality for which the actions bear significance. Through this process of appraisal, it is then possible to configure the manner in which the relation between self and goal is articulated. Among key physical determinants influencing the linkage of bodily representation to the individual, for example, are thermodynamic realities governing organismal dynamics. Prigogine notably showed that living systems were open to energy exchange, thus delimiting the whole organism as a domain of energy flux, with its topological perimeters defining the boundaries for systemic constraints. As a corollary the global organizational network of the organism maintains a continual 
cycle of organismal action and reconstitution, which is subject to global energy needs. Within a thermodynamic context, accordingly, discrete actions clearly have repercussions for the energy requirements of the entire body [19].

\section{Referencing Dynamic Actions to the Body}

Crucially, the unitary nature of the self is dictated by the body's dynamism. At any moment in time, the body's complex dynamics emerge from a just occupied spatiotemporal domain to enter a newly present, newly configured zone [5]. Temporally fused at this evolving junction are the spatial and temporal coordinates of the corporal entity from which discrete limb motions emerge. Thus, at each moment in time the body's spatial configuration constitutes a new source of stability that references ongoing, goal oriented motor activity. Because this junction entails the whole corpus this transition everywhere engages neural representations that link processes of the body trajectory at multiple scales [15], in widely variant neural locations, and with continually varying emphasis. As an 'interactive unit' in an environmental space the continual demand for sensory assessment requires the updating of these representations to maintain a holistic representation despite a continually evolving stability-flexibility junction.

Existing evidence indicates that this unitary dynamism is mediated chiefly through reciprocal contributions emerging from the neural activity of body and brain. Its generation is due to two separately staged and temporally disparate neural processes. In the first the reciprocal influences enhance activity dependent plastic integration at the synaptic level, which shapes the circuits receiving and interpreting sensory imagery. That is, it structures the synaptic organization to be responsive to 'real time' dynamic activity by plastically molding a functional synergy between body and brain, which dynamically regulates the cortical reception of information that has been channeled inward. Neuroscientific studies show, for example, that light stimulation configures ocular dominance columns in the occipital cortex [20] and that somato-topic input shapes hippocampal memory circuits for topographical mapping during navigational exploration [21]. Interestingly, sensory reception is delimited by siting it to a unique bodily mooring; accordingly, sensory reception constructs the brain's perception of the world in terms of the body [22]. In fact, the choice of sensory modalities defines the transmission of sensory input, controlling information input that shapes the brain [23], vividly demonstrated in cases of lost limbs where molding influences of limbs persist, often for years. Their siting to the body's topological perimeter is intuitively significant since this, rather than the brain's cortical structure, is the point where the body intersects the world; hence, responses to events in the world are determined by the body's configuration.

In the second, the complementary contributions from brain and body exploit this configured neural architecture to construct a unitary, performance dynamic where the individual is both perceived and actively engaged with the world. In Varela, Thompson, and Rosch's conception [10], this unites perception and action -'By using the term action we mean to emphasize once again that sensory and motor processes, perception and action, are fundamentally inseparable in lived cognition' - underscoring the reality of the unity between perception and individual action.

As a dynamic reality the perception action loop is closely related to the forward projection model of the motor image, which encompasses both action identification and its association with the bodily representation. In primitive organisms, like C elegans, that lack the motor image both elements are linked together through the global neural representation of the organism [24]. This enables the organisms to differentiate the physical space subsumed by processes belonging to itself from those of the contiguous environment. This range has been shown to be contained in representational content characterized by attractor dynamics that relate to the entire organism. Representational content for the discrete movements in turn are subsumed to the global representation when the animals engage in motor activity.

How global representations track bodily configurations across an evolving spatial and temporal panorama at multiple scales and amid a continually evolving context is nonetheless uncertain in the more complex advanced species that employ the motor image; however, current work is beginning to disentangle representational content for discrete limb movements from that more globally attuned to the whole individual. These studies suggest that representations of body posture are likely to be an important proxy for global self-representation in dynamic actions [15]. Intuitively, this is apparent for several reasons. For one thing, body posture entails the whole corpus at any moment in time. The phenomenological philosopher Merleau Ponty, for example, uses the term the lived body to describe the habitual resource for expression offered by the body's dynamic whole. For another, it is as a holistic dynamic that the individual actively interacts with the world through time; hence, unified representations are needed to track the evolving spatial and temporal circumstances of this holistic engagement.

Because motor planning is referenced to the body, however A not B experiments, for example, reveal that effector actions are embedded in bodily activity frameworks - isolation of effector representations is complicated by cross correlation between activity associated with many aspects of movement. In arm reaching, for example, representations of limb movement, target, and body are all correlated in the linear decoding methods required for analysis; accordingly the motion elements need to be decorrelated. Such decorrelation strategies for individual feature elements have been informative for distinguishing discrete movement elements from the combined neural activity. For example, target timing properties can be used to decode target direction independently from initial hand direction in the planning period [25].

Elementary motor representations, for instance, are now known to be assembled in forward projecting, motion models that are cognitively employed to control rapid movements [26]. Unlike forward efference copies that directly anticipate sensory input of slow movements, projection models for rapid movements need to additionally embed the efference copy within a projected forward model of expected system dynamics in order to overcome time delays of sensory feedback and allow for accurate positioning 
assessments for needed corrections. Neurons encoding forward angle estimation have, in fact, been localized to the posterior parietal cortex (PPC). Their siting to the PPC suggests, further, that it functions as a hub in integrating discrete effector movement within a larger bodily dynamic [27]. These effective functional connectivities, moreover, are likely to extend to the premotor cortices, since studies of grasping neurons show that coding of simple action goals entails the additional involvement of premotor cortical regions.

Insight into how these elements are assembled into the more complex representations of the motor image has come from studies focusing on transformation of coordinate systems between the eye and the limb and body. Visually guided limb movements, for example, require that eye coordinates be transformed to limb and body centered ones. A likely first step in this transformation is the modulation of eye centered coordinates by head position signals, termed gain field influences [28]. Since the ventral premotor cortex codes in body centered coordinates, contributions from the posterior parietal cortex and the premotor cortex again appear likely loci for referencing individual effector motions within the global dynamical framework. Consistent with this interpretation posture-related neuronal coding for head, back, and neck are detected in freely moving rats. For instance, recordings from cells in the PPC uniquely respond to conjunctive postures to head and back or to whole body configurations [15].

\section{Modulating Dynamic Representations of Self}

Intuitively, motor planning is understood to be formulated for specific goals that are relevant for the individual. Converging evidence obtained from schizophrenia patients, for example, suggests that bodily representations are uniquely modified by goal directed content. Notably, disturbances in the sense of self that mark schizophrenia in its prodromal and acute stages have been the basis for the classification of the loss of self as a core symptom [29]. This consensus is based on neurocognitive and psychopathological evidence, in which both body ownership and personal agency are impacted, and where affected individuals exhibit distinct neural signatures [30] involving effective connectivities [31]. Normal patients show a consistently high correspondence between structural networks from fMRI modules and those from diffusion imaging, across a wide range of populations. Patients with child onset schizophrenia, by contrast, display significantly different modular communities than normal individuals, particularly in the right insular and perisylvian regions.

How the representation of self is affected in these patients and how it is linked to the body has been suggested in experiments designed to evoke misattribution errors. Whereas normal individuals display error rates of 20 to 30 percent in such experiments, schizophrenia patients show rates as high as 80 percent. In a majority of the cases the patient misattributes the motion of the alien hand to himself. On the basis of such results, in fact Frith [31] proposed that deficient self-monitoring is greatly exaggerated in the schizophrenic patient.

Since the whole body is used to anchor the self-representation in both automatic predictive processing and in intentional goal directed activity, however, the question of how the representational content of the whole body is differentiated in automatic vs intentional activity is left unresolved. Subsequent experiments designed to resolve this uncertainty have shown that these patients automatically adapt their movements in visuomotor conflicts, reaching their targets despite distorted visual feedback. That is, they are unimpaired in automatically adjusting their performance, incorporating them in efficient and coordinated motor strategies. On the other hand, when schizophrenia patients become consciously aware of the experimentally imposed conflict, they are unable to correct their motions. In other words, schizophrenia patients appear to lack a capacity to consciously associate their self-initiated motions to a representation of the self. As the wholebody representation does not itself appear to be impaired patients automatically make predictive and prudential inferences on the basis of the whole body - the mechanisms associated with linking intentions to bodily representation, instead, appear to be affected. Taken together, the inability of schizophrenia patients to consciously attribute bodily actions to the self-reveals a failure to link the intentions and desires of a protagonist to an intact representation of the body.

One outcome predicted by this understanding is that the representational content of the self-varies as a function of the specific goals consciously embraced by the author of those intentions. As a corollary, schizophrenia patients ought to be deficient in inscribing goal specific features within the self-representation; that is, the representation of the self in such patients will not only differ with respect to the self-content of normal individuals but also with respect to the self-content of different goal contexts. This prediction is briefly considered here in two cases of intentional actions that substantially differ with respect to the nature of the goal for which the actions are willed, self/other social interactions and action attribution. As a class, social interactions are unique for their requirement to carefully delineate the self from the subjective features that characterize other individuals $[32,33]$. Research from social psychology indicates that the self-representation is not only unique to the individual but also shares common features that assist in identifying others like himself. Developmental processes for self-other perceptions, for example, entail two stages, one where the self-percept is configured first by the association with the body, and also a second in which the self-construct is elicited by intersubjectively through a Lacan style identification [34]. Indeed, social neuroscience has revealed a broad cluster of dedicated cells and circuits that are used to distinguish self from other, now classed under theory of mind. As a proxy for self-other interactions these distinctions recognize the spatial domain surrounding the body as the space uniquely belonging to the self [35]. Though this domain is amenable to manipulation its range is nonetheless restricted, circumscribing a domain beyond which the self is no longer perceived; accordingly, this reveals its dependence on bodily content for its configuration. Significantly, the ability to situate the inhabited space of the self, is substantially altered in schizophrenia subjects, who display weak and shallow personal spaces in which the self is not easily differentiated from the other [36].

By way of contrast, goal directed actions that entail limb movements alone do not require the clear demarcation between 
self and other that characterize social interactions. In such cases, the bodily representation is instead modified to incorporate the actions that have as their outcome an intended goal terminus that engages bodily activities; here, the author's intentions clearly differ from those evoked in social interaction. Accordingly, when the motor trajectory entails, for example, the intentional use of a utensil the self representation is correspondingly modified, a coincidence not evidenced in schizophrenia patients [37].

Taken together, these, and Jeannerod's observation [7] of two levels of self recognition, reveal a close correspondence between goal directed information and the self representation, suggesting that the self representation is directly modified by goal specific content. Mechanisms that are likely to shape self content can therefore be expected to include cells, circuits, or processes that bear desires and intentions of the author. One likely origin of such information is that contained in egocentric networks. These networks encode unique, agent specific content about an experience [38]. Egocentric networks, for example, enframe content by relating an individual to objective, representational content of an experience. Allocentric networks, by contrast, contain spatial information that provides only objective details about an experience.

Evidence localizing egocentric frameworks, site these to specific domains of the hippocampus, such as the lateral entorhinal cortex [38], and to the angular gyrus of the parietal cortex $[39,40]$, a region that has been previously identified with self and bodily representation. Goal directed information contained in these networks thus can be expected to uniquely modify the self representation by relating the individual to a intended terminus via information that is goal specific. Consistent with this expectation imaging and electrophysiological experiments of grasping motions show that neurons of parietal and premotor areas exhibit not only coding of goal content but its dependence on consciously retaining the intention. Recordings from these neurons specifically evidence action goal selectivity through tuning to sensorial information as it becomes available, or in late action sequence phases when only the memory of the intention is present. Significantly, when such informational content is prevented, tuning disappears.

\section{Conclusion and Summary}

Autonomous activity marks the living organism as a 'unit of interactivity', understood as an agent independently pursuing a plethora of goals in an interactive space. Neural mechanisms enabling such goal specific pursuit have to date remained unidentified, but are nonetheless intuitively evident in behaviors that link the individual as a dynamic and holistic reality to a designate objective. Consistent with this intuition, experimental observations reveal that neural representations of bodily posture are a likely neural substrate for self content that is uniquely modified according to the goal that is chosen.

\section{Acknowledgement}

None.

\section{Conflict of Interest}

No conflict of interest.

\section{References}

1. Jeannerod M (2005) Levels of representation of goal-directed actions. In: HJ Fruend, M Jeannerod, M Hallett, R Leiguarda (eds.) Higher-order Motor Disorders. Oxford University Press, Oxford, UK.

2. Lashley KS (1951) The problem of serial order in behavior. In: LA Jeffress (ed.) Cerebral Mechanisms and Behavior. Wiley Press, New York, USA.

3. Held R (1961) Exposure-history as a factor in maintaining stability of perception and coordination. J Nerv Ment Dis 132: 26-32.

4. Frak V, Nazir T, Goyette M, Cohen H, Jeannerod M (2010) Grip force is part of the semantic representation of manual action verbs. PLoS One 5(3): 9728.

5. Smith L (2009) Stability and flexibility in development. In: Spencer J, Thomas MSC, McClelland JL, (eds.) Toward a Unified Theory of Development. Oxford University Press, Oxford, UK.

6. Corbetta D (2009) Brain, body, and mind: lessons from infant motor development. In: Spencer J, Thomas MSC, McClelland JL (eds.) Toward a Unified Theory of Development. Oxford University Press, Oxford, UK.

7. Jeannerod M (2009) The sense of agency and its disturbances in schizophrenia: a reappraisal. Exp Brain Res 192(3): 527-532.

8. Von Holst E, Mittelstaedt H (1950) The reafference principle. Interactions between central nervous system and periphery. Natural Sciences 37: 464-476.

9. Bizzi E, Kalil RE, Tagliasco V (1971) Eye head coordination in monkeys: evidence for centrally patterned organization. Science 173 (3995): 452454.

10. Varela F, Thompson E, Rosch E (1991) The embodied mind: cognitive science and human experience. Cambridge, MA: MIT Press, USA.

11. Campos JJ, Bertenthal BI, Kermoian R (1992) Early experience and emotional development: the emergence of wariness of heights. Psychol Sci 3:61-64.

12. Bushnell EW, Boudreau JP (1993) Motor development and the mind: the potential role of motor abilities as a determinant of aspects of perceptual development. Child Dev 64: 1005-1021.

13. Lockman JJ, Thelen E (1993) Developmental biodynamics: brain, body, behavior connections. Child Dev 64(4): 953-959.

14. Larrivee D (in press) Realigning the Neural Paradigm for Death J Bioethical Inquiry.

15. Mimica B, Dunn BA, Tombaz T, Bojja VP, Whitlock JR (2018) Efficient cortical coding of 3D posture in freely behaving rats. Science 362(6414): 584-589.

16. Fourneret P, de Vignemont F, Franck N, Slachevsky A, Dubois B, et al. (2002) Perception of self-generated action in schizophrenia. Cogn Neuropsychiatry 7(2): 139-156.

17. Hur JW, Kwon JS, Lee TY, Park S (2014) The crisis of minimal selfawareness in schizophrenia: a meta-analytic. Schizophr Res 152(1): 5864.

18. Hecht D (2010) Schizophrenia, the sense of 'self' and the right cerebral self' and the right cerebral hemisphere. Med Hypotheses 74(1): 186188.

19. Allen M, Friston KJ (2018) From cognitivism to autopoiesis: towards a computational framework for the embodied mind. Synthese 195: 24592482.

20. Wiesel and Hubel Wiesel TN, Hubel DH (1963) Single cell responses in striate cortex of kittens deprived of vision in one eye. J Neurophys 26: 1003-1017.

21. Moser MB, Moser EI, Forrest E, Andersen P, Morris RGM (1995) Spatial learning with a minislab in the dorsal hippocampus. Proc Natl Acad Sci 92(21): 9697-9701.

22. Gallagher S (2005) How the body shapes the mind. Oxford: Oxford University Press, UK. 
23. Moya P (2014) Habit and embodiment in Merleau-Ponty. Frontiers Human Neurosci 8: 542.

24. Kato S, Kaplan HS, Schrodel T, Skora S, Lindsay TH et al. (2015) Global brain dynamics embed the motor command sequence of Caenorhabditis elegans. Cell 163: 656-669.

25. Pearce TM, Moran DW (2012) Strategy dependent encoding of planned arm movements in the dorsal premotor cortex. Science 337(6097): 984989.

26. Mulliken GH, Musallam S, Andersen RA (2008) Forward estimation of movement state in posterior parietal cortex. Proc Nat Acad Sci 105(24): 8170-8177.

27. Anderson RA (1997) Multimodal integration for the representation of space in the posterior parietal cortex. Phil Trans R Soc Lond B 352:1421 1428.

28. Brozovic M, Gail A, Andersen RA (2008) Mechanisms of gail modulation at single neuron and network levels. J Comput Neurosci 25(1): 158-168.

29. Ferri F, Frassinetti F, Mastrangelo F, Salone A, Ferro FM, et al. (2012) Bodily self and schizophrenia: the loss of implicit self-body knowledge. Conscious Cogn 21(3): 1365-1374.

30. Bloch A, Lambiotte R, Roberts B, Giedd J, Gogtay N, et al. (2012) The discovery of population differences in network community structure: new methods and applications to brain functional networks in schizophrenia. Neuroimage 59: 3889-3900.

31. Frith C (2012) Explaining delusions of control: the comparator model 20 years on. Conscious Cogn 21(1): 52-54.
32. Decety J, Sommerville JA (2003) Shared representations between self and other: a social cognitive neuroscience view. Trends Cogn Sci 7(12): 527-533.

33. Decety J, Lamm C (2007) The role of the right temporoparietal junction in social interaction: how low-level computational processes contribute to metacognition. The Neuroscientist 13(6): 580-593.

34. Dumas G, Kelso JAS, Nadel J (2014) Tackling the social cognition paradox through multi-scale approaches. Front Psychol 5: 882.

35. Noel JP, Pfeiffer C, Blanke 0, Serino A (2015) Peripersonal space as the space of the bodily self. Cognition 144: 49-57.

36. Noel JP, Cascio CJ, Wallace MT, Park S (2017) The spatial self in schizophrenia and autism spectrum disorder. Schizophrenia Res 179: 8-12.

37. Orban GA, Rizzolatti G (2012) An area specifically devoted to tool use in human left inferior parietal lobule. Behav Brain Sci 35(4): 234.

38. Wang C, Chen X, Lee H, Deshmukh SS, Yoganarasimha D, et al (2018) Egocentric coding of external items in the lateral entorhinal cortex. Science 362(6417): 945-949.

39. Bonnici HM, Cheke LG, Green DAE, FitzGerald HMB, Simons JS (2018) Specifying a causal role for angular gyrus in autobiographical memory. J Neurosci 38(49): 10438-10443.

40. Bonini L, Serventi FU, Simone L, Rozzi S, Ferrari PF, et al. (2011) Grasping neurons of monkey parietal and premotor cortices encode action goals at distinct levels of abstraction during complex action sequences. Neurosci 31(15): 5876-5887. 